\title{
Elevated Risk of an Intermediate or High SYNTAX Score in Subjects with Impaired Fasting Glucose
}

\author{
Xishan Yang ${ }^{1}$, Hui Liu ${ }^{2}$, Fangfang Yang ${ }^{1}$, Pingshuan Dong ${ }^{1}$, Xianen $\mathrm{Fa}^{3}$, Qingyong Zhang ${ }^{3}$, \\ $\mathrm{Li} \mathrm{Li}^{4}$, Zhikuan Wang ${ }^{4}$ and Di Zhao ${ }^{4}$
}

\begin{abstract}
Objective This study was designed to determine the SYNTAX score under different fasting plasma glucose (FPG) states in Chinese patients undergoing coronary angiography, particularly subjects with impaired FPG.

Methods Four hundred and forty-six subjects undergoing coronary angiography were enrolled in this study and divided into four groups based on the FPG level or a history of type 2 diabetes mellitus (T2DM): normal FPG, impaired FPG, known and previously unknown T2DM.

Results The angiographic SYNTAX scores were higher in the subjects with known $(\mathrm{p}<0.001)$ or previously unknown $(\mathrm{p}<0.001)$ T2DM than in those with normal FPG. There were significant differences in the number of diseased coronary artery vessels between the subjects with known $(\mathrm{p}<0.01)$ or unknown T2DM $(\mathrm{p}<0.05)$ and the subjects with normal FPG. However, there were no significant differences in the SYNTAX score or the number of diseased coronary artery vessels between the subjects with impaired FPG and those with normal FPG. The subjects with impaired FPG (2.917-fold, $\mathrm{p}=0.004)$ and known (3.064-fold, $\mathrm{p}=0.000)$ or previously unknown (3.301-fold, $\mathrm{p}=0.000$ ) T2DM exhibited a significantly elevated risk of having an intermediate or high SYNTAX score compared with the subjects with normal FPG.

Conclusion Chinese subjects with impaired FPG have a significantly elevated risk of having an intermediate or high SYNTAX score, indicating a risk of severe coronary artery lesions. Subjects with known or previously unknown T2DM may have severe coronary artery lesions. These findings suggest the importance of achieving better glycemic control in order to prevent coronary atherosclerosis and improve the cardiovascular prognosis.
\end{abstract}

Key words: fasting plasma glucose, coronary artery disease, SYNTAX score

(Intern Med 54: 439-444, 2015)

(DOI: 10.2169/internalmedicine.54.3295)

\section{Introduction}

It is well known that type 2 diabetes mellitus (T2DM) is a particularly high risk factor for cardiovascular disease (CVD) (1) and that CVD is the leading cause of death among patients with T2DM (2). Many individuals with prediabetes, which refers to impaired fasting glucose, impaired glucose tolerance or both, already display microvascular disease consequences similar to those seen in patients with T2DM (3). However, it is not established whether prediabe- tes should be considered a coronary heart disease risk equivalent (3), and there are controversial data concerning the correlation between a blood glucose level in the subdiabetic range (impaired fasting glucose and impaired glucose tolerance) and cardiovascular risks (4). Several studies have shown that the fasting plasma glucose (FPG) level is related to the severity and extent of coronary artery lesions (5-7). The SYNTAX score is determined based on the qualitative and quantitative characterization of coronary artery disease (CAD), including 11 angiographic variables that take into consideration the lesion location and characteris-

\footnotetext{
${ }^{1}$ Division of Cardiology, the First Hospital Affiliated to Henan Science and Technology University, China, ${ }^{2}$ Division of Endocrinology, Luoyang Central Hospital Affiliated to Zhengzhou University, China, ${ }^{3}$ Division of Cardiothoracic Surgery, the Second Hospital Affiliated to Zhengzhou University, China and ${ }^{4}$ Division of Hypertension, the Second Hospital Affiliated to Zhengzhou University, China

Received for publication May 15, 2014; Accepted for publication July 21, 2014

Correspondence to Dr. Di Zhao, emaildizhao@yahoo.com
} 
Table 1. Subject Characteristics

\begin{tabular}{|c|c|c|c|c|c|}
\hline & $\begin{array}{l}\text { Normal } \\
\text { FPG }\end{array}$ & $\begin{array}{l}\text { Impaired } \\
\text { FPG }\end{array}$ & $\begin{array}{l}\text { Known } \\
\text { T2DM }\end{array}$ & $\begin{array}{l}\text { Unknown } \\
\text { T2DM }\end{array}$ & $\mathrm{p}$ value \\
\hline & $\mathrm{n}=212$ & $\mathrm{n}=84$ & $\mathrm{n}=85$ & $\mathrm{n}=65$ & \\
\hline Age (years) & $60 \pm 0$ & $61 \pm 1$ & $62 \pm 1$ & $60 \pm 1$ & 0.20 \\
\hline Male/Female (n) & $142 / 70$ & $59 / 25$ & $40 / 45 * *$ & $44 / 21$ & 0.004 \\
\hline $\begin{array}{l}\text { Smoker/Non } \\
\text {-smoker (n) }\end{array}$ & $77 / 135$ & $35 / 49$ & $22 / 63$ & $27 / 38$ & 0.12 \\
\hline $\mathrm{WC}(\mathrm{cm})$ & $90.4 \pm 0.4$ & $90.9 \pm 0.6$ & $89.8 \pm 0.6$ & $89.3 \pm 0.8$ & 0.28 \\
\hline Body Weight (kg) & $69.7 \pm 0.7$ & $70.6 \pm 1.1$ & $68.6 \pm 1.0$ & $67.7 \pm 1.4$ & 0.33 \\
\hline BMI $\left(\mathrm{kg} / \mathrm{m}^{2}\right)$ & $25.3 \pm 0.2$ & $25.6 \pm 0.3$ & $24.8 \pm 0.3$ & $24.6 \pm 0.3$ & 0.08 \\
\hline SBP (mm Hg) & $128 \pm 2$ & $127 \pm 2$ & $128 \pm 3$ & $127 \pm 3$ & 0.99 \\
\hline $\mathrm{DBP}(\mathrm{mm} \mathrm{Hg})$ & $78 \pm 1$ & $79 \pm 1$ & $77 \pm 1$ & $82 \pm 2$ & 0.10 \\
\hline $\mathrm{FPG}(\mathrm{mmol} / \mathrm{L})$ & $4.5 \pm 0.0$ & $6.1 \pm 0.1 * * *$ & $8.2 \pm 0.4 * * *$ & $9.2 \pm 0.4 * * *$ & 0.0001 \\
\hline HbAlc $(\%)$ & $5.0 \pm 0.1$ & $5.8 \pm 0.2 * * *$ & $8.3 \pm 0.2 * * *$ & $8.6 \pm 0.2 * * *$ & 0.0001 \\
\hline HOMA-IR & $1.2 \pm 0.1$ & $1.8 \pm 0.2 * *$ & $2.7 \pm 0.2 * * *$ & $3.2 \pm 0.3 * * *$ & 0.0001 \\
\hline $\mathrm{TC}(\mathrm{mmol} / \mathrm{L})$ & $4.50 \pm 0.07$ & $4.65 \pm 0.11$ & $4.69 \pm 0.14$ & $4.74 \pm 0.13$ & 0.27 \\
\hline LDL-C $(\mathrm{mmol} / \mathrm{L})$ & $2.58 \pm 0.07$ & $2.06 \pm 0.10$ & $2.50 \pm 0.13$ & $2.47 \pm 0.13$ & 0.78 \\
\hline HDL-C (mmol/L) & $1.09 \pm 0.02$ & $1.06 \pm 0.03$ & $1.04 \pm 0.04$ & $1.10 \pm 0.04$ & 0.59 \\
\hline $\mathrm{TG}(\mathrm{mmol} / \mathrm{L})$ & $1.55 \pm 0.07$ & $1.78 \pm 0.14$ & $1.89 \pm 0.13$ & $1.74 \pm 0.21$ & 0.12 \\
\hline UA (mg/dL) & $305 \pm 6$ & $293 \pm 10$ & $278 \pm 10$ & $278 \pm 11$ & 0.05 \\
\hline $\begin{array}{l}\text { eGFR } \\
\left(\mathrm{mL} / \mathrm{min} / 1.73 \mathrm{~m}^{2}\right)\end{array}$ & $102.8 \pm 2.5$ & $95.0 \pm 4.7$ & $95.5 \pm 4.6$ & $89.4 \pm 3.2$ & 0.06 \\
\hline $\begin{array}{l}\text { Family History } \\
\text { of CVD }(\%)\end{array}$ & 28.3 & 27.4 & 23.5 & 24.6 & 0.51 \\
\hline \multicolumn{6}{|c|}{$\begin{array}{l}\text { As compared with subjects with normal fasting plasma glucose }(\mathrm{FPG}), * *: \mathrm{p}<0.01 \text {, } \\
* * * \text { : }<0.001 \text {. T2DM: type } 2 \text { diabetes mellitus, WC: waist circumference, BMI: body } \\
\text { mass index, SBP: systolic blood pressure, DBP: diastolic blood pressure, HbA1c: } \\
\text { glycated hemoglobin A1c, HOMA-IR: homeostatic model assessment of insulin } \\
\text { resistance, TC: total cholesterol, LDL-C: low density lipoprotein cholesterol, HDL-C: } \\
\text { high density lipoprotein cholesterol, TG: triglyceride, UA: uric acid, eGFR: estimated } \\
\text { glumerular filtration rate, CVD: cardiovascular disease }\end{array}$} \\
\hline
\end{tabular}

tics (8). This score has been shown to be an effective tool for risk stratifying patients with complex CAD undergoing percutaneous coronary intervention in the landmark SYNTAX trial as well as other clinical settings (8). At present, however, the relationship between FPG and the SYNTAX score remains unclear in subjects undergoing coronary angiography, especially those with impaired FPG.

Our hypothesis is that FPG is associated with the severity and extent of coronary artery lesions assessed according to the SYNTAX score in subjects with impaired FPG and/or known or previously unknown T2DM. Therefore, this study was designed to determine the SYNTAX score under different fasting glucose states in subjects undergoing coronary angiography. We also analyzed the risk of severe coronary artery lesions in subjects with impaired FPG and/or known or previously unknown T2DM.

\section{Materials and Methods}

\section{Subjects}

Four hundred and forty-six subjects undergoing coronary angiography were enrolled in this study. T2DM was defined as an elevated fasting blood glucose (FBG) level $(\geq 7.0$ $\mathrm{mmol} / \mathrm{L}$ ) or hemoglobin A1c value (HbA1c $\geq 6.5 \%, 48$ $\mathrm{mmol} / \mathrm{moL}$ ). Impaired FPG was defined as $5.6 \leq \mathrm{FPG}<7.0$ $\mathrm{mmol} / \mathrm{L}$ (3). The subject characteristics are shown in Table 1. Patients with a history of previous percutaneous coronary intervention, coronary artery bypass grafting, acute myocardial infarction, severe liver or renal disorders and malignancy were excluded. This study was approved and written consent was obtained from the Institutional Review Boards at the First Hospital Affiliated to Henan Science and Technology University, Luoyang Central Hospital Affiliated to Zhengzhou University and Second Hospital Affiliated to Zhengzhou University. All participants provided their written informed consent to participate in this study. Systolic blood pressure (SBP), diastolic blood pressure (DBP), FPG, blood lipids and several biochemical parameters were determined. Drugs used to treat hypertension, T2DM and dyslipidemia were continued in this study.

\section{Coronary artery angiography and the SYNTAX score}

Coronary angiography was performed according to the Judkins technique via radial artery access. The angiographic characteristics, including the lesion location and percent stenosis, of all coronary lesions on the index coronary angiogram were obtained by thoroughly reviewing each angiogram. CAD was defined as a luminal diameter stenosis of $>50 \%$ in at least one major epicardial coronary artery. The SYNTAX scoring system was used to calculate the coronary disease severity. The grade of luminal narrowing was determined according to the consensus opinion of two experienced interventional cardiologists. The standards for the SYNTAX score are as follows (9): Low: 0-22, Intermediate: 23-32, High: 33 or more. 
A

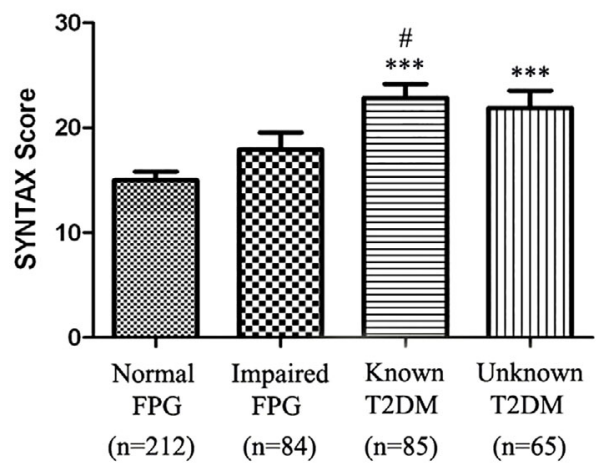

B

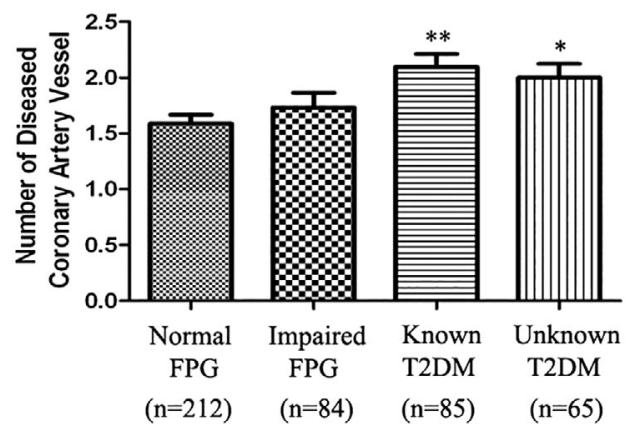

Figure. Comparison of the SYNTAX scores and number of diseased coronary artery vessels in the subjects with different FPG states undergoing coronary angiography. As compared with the subjects with normal FPG, *: p<0.05, **: p<0.01, ***: $\mathbf{p}<0.001$. As compared with the subjects with impaired FPG, \#, p<0.05. FPG: fasting plasma glucose, T2DM: type 2 diabetes mellitus, SYNTAX score: a system for calculating coronary disease severity using angiography

\section{Biochemical determination}

Three milliliters of blood was collected after 12 hours of fasting and then centrifuged at 3,000 revolutions per minute for 10 minutes. The plasma was snap-frozen and stored at $-20^{\circ} \mathrm{C}$ for the measurements. The FPG and serum lipids were measured on an automatic biochemical analyzer (Hitachi H7600, Hitachi, Tokyo, Japan). The HbA1c level was measured using an immunoturbidimetric assay with the Hitachi H7600. The homeostatic model assessment of insulin resistance (HOMA-IR) was calculated as follows: [fasting plasma insulin $(\mathrm{FPI})(\mathrm{mU} / \mathrm{L}) \times \mathrm{FPG}(\mathrm{mmol} / \mathrm{L})] / 22.5(10)$. The estimated glomerular filtration rate (eGFR) was calculated according to the modification of diet in renal disease (MDRD) equation: eGFR $=186 \times$ serum creatinine $(\mathrm{mg} / \mathrm{dL})$ - 1.154xage (years) - 0.203 (0.742, if female) (11).

\section{Statistical analysis}

The statistical analysis was performed using the GraphPad PRISM program (Prism 5, GraphPad, San Diego, USA) and SPSS version 17.0 (SPSS Incorporated, Chicago, USA) software packages. Four-group comparisons were performed using a one way analysis of variance (ANOVA) with a post hoc test of Newman-Keuls multiple comparisons for twogroup comparisons. Ratio comparisons were made using the Chi-square test. The adjusted odds ratio was analyzed ac- cording to a logistic regression analysis with the SPSS software packages. The results are presented as the mean \pm SE. The level of significance was set at a $\mathrm{p}$ value of $<0.05$.

\section{Results}

\section{Comparison of subject characteristics}

The subject characteristics are shown in Table 1. The male/female ratio was lower in the subjects with known T2DM than in the subjects with normal FPG $(\mathrm{p}<0.01)$. There were significant differences in the FPG, HbAlc and HOMA-IR values among the subjects with formal FPG, impaired FPG and known or previously unknown T2DM (all $\mathrm{p}$ $<0.001)$. However, there were no significant differences in waist circumference (WC), SBP, DBP, high-density lipoprotein-cholesterol (HDL-C) or triglycerides (TG) among these groups ( $>0.05)$.

\section{Comparison of the SYNTAX score and number of diseased coronary artery vessels under different fasting glucose states}

As shown in Figure A, the angiographic SYNTAX scores were higher in the subjects with known $(\mathrm{p}<0.001)$ or previously unknown $(\mathrm{p}<0.001)$ T2DM than in the subjects with normal FPG. However, there were no significant differences in the angiographic SYNTAX scores between the subjects with impaired FPG and normal FPG ( $>>0.05)$. As shown in Figure $\mathrm{B}$, there were significant differences in the number of diseased coronary artery vessels between the subjects with known $(\mathrm{p}<0.01)$ or previously unknown T2DM $(\mathrm{p}<0.05)$ and the subjects with normal FPG. In contrast, there were no significant differences in the number of diseased coronary artery vessels between the subjects with impaired FPG and those with normal FPG $(\mathrm{p}>0.05)$.

\section{Adjusted odds ratio of an intermediate or high SYN- TAX score under different fasting glucose states}

As shown in Table 2, the subjects with impaired FPG (2.917-fold, $\mathrm{p}=0.004$ ) and known (3.064-fold, $\mathrm{p}=0.000$ ) or previously unknown (3.301-fold, $\mathrm{p}=0.000) \mathrm{T} 2 \mathrm{DM}$ exhibited a significantly elevated risk of having an intermediate or high SYNTAX score compared with those with normal FPG.

\section{Discussion}

In the present study, we provide the evidence that Chinese subjects with impaired FPG have a significantly elevated risk of having an intermediate or high SYNTAX score. Subjects with known or previously unknown T2DM may have severe coronary artery lesions, as assessed according to the SYNTAX score. These findings suggest the importance of achieving better glycemic control in order to prevent coronary atherosclerosis and improve the cardiovascular prognosis. Interestingly, we originally analyzed our data based on 
Table 2. Adjusted Odds Ratio for Prediction of Intermediate and High SYNTAX Score according to FPG

\begin{tabular}{lccc}
\hline FPG & Adjusted Odds Ratio & $95 \% \mathrm{CI}$ & $\mathrm{p}$ value \\
\hline Normal FPG & 1.00 (Reference) & \\
Impaired FPG & 2.917 & $1.421-5.988$ & 0.004 \\
Known T2DM & 3.064 & $1.905-4.927$ & 0.000 \\
Unknown T2DM & 3.301 & $1.769-6.163$ & 0.000 \\
\hline T2DM: type 2 diabetes mellitus, FPG: fasting plasma glucose. SYNTAX score: a \\
system to assess severity and extent of coronary artery lesions. The standard are as \\
following. Low: 0-22, Intermediate: 23-32, High: 33 or more
\end{tabular}

the criterion for impaired FPG $(6.1 \leq$ FPG $<7.0 \mathrm{mmol} / \mathrm{L})$, the present criterion used in China, and found that the SYNTAX scores in the subjects with impaired FPG were significantly higher than those in the subjects with normal FPG. However, $5.6 \leq \mathrm{FPG}<7.0 \mathrm{mmol} / \mathrm{L}$ is the updated criterion for impaired FPG in several countries. Considering comparisons with data obtained by other scholars, we decided to use the updated criterion for impaired FPG.

Although diabetes increases the risk of CVD and mortality, the dose-response relationship between a fasting glucose level below that diagnostic for diabetes with cardiovascular events has not been well characterized (12). Impaired fasting glucose should be considered a predictor of the risk of CAD (12) and Shaye et al. (13) found that an elevated CVD risk is strongly and independently associated with a glucose level within the normoglycemic range. In the Reykjavik Study and a meta-analysis of other Western prospective studies, the fasting and post-load glucose levels were found to be modestly associated with coronary heart disease risk in subjects without diabetes (14). Japanese adults with impaired glucose tolerance or impaired fasting glucose have also been shown to have an increased risk of CAD events (15). Furthermore, Schinner et al. (4) found a continuously increasing risk of coronary heart disease associated with fasting and 2-hour post-load blood glucose levels in the sub-diabetic glucose range, although no clear cut-off values for cardiovascular risk were determined. Meanwhile, Bhat et al. (16) reported that the greater the FPG concentration, the more adverse the coronary heart disease risk profile. Therefore, assessing the FPG level may help to identify apparently healthy persons with early metabolic abnormalities who are at increased risk of CVD before they progress to prediabetes or overt diabetes mellitus. Moreover, Laukkanen et al. (17) found that impaired FPG and T2DM represent risk factors for sudden cardiac death, and the exercise capacity in patients with CAD is related to the FBG level (18). In contrast, Arbel et al. (19) reported no correlations between the admission glucose or fasting glucose levels and the severity of CAD in non-diabetic patients with myocardial infarction or stable angina, whereas the HbAlc level, the only glucometabolic factor associated with the SYNTAX score, is significantly associated with CAD severity. A meta-analysis also suggested a somewhat stronger association between the HbA1c level and the risk of coronary heart disease (14). Interestingly, the FPG level has been reported to perform bet- ter than the HbA1c level in screening for diabetes among patients undergoing coronary artery angiography (20). Oral glucose tolerance tests should be routinely performed to diagnose diabetes in patients with CAD undergoing coronary angiography, as HbAlc measurement alone appears to miss a substantial proportion of patients with silent diabetes (21). In the present study, a significantly elevated risk of having an intermediate or high SYNTAX score was observed in the subjects with impaired FPG compared with that noted in the subjects with normal FPG, although we failed to detect higher SYNTAX scores in the subjects with impaired FPG. Furthermore, both known and previously unknown T2DM were associated with a higher SYNTAX score and carried a significantly elevated risk of having an intermediate or high SYNTAX score compared with that observed in the subjects with normal FPG. These findings indicate the presence of a dose-response relationship between FPG and severe coronary artery lesions. Berry et al. (6) reported that the fasting blood glucose and HbA1c levels and presence of diabetes were associated with the severity and progression of coronary atherosclerosis. These observations support the hypothesis that achieving better glycemic control may favorably influence the disease course of CAD in patients with abnormal glucose tolerance or diabetes (6). Basic vascular biology data as well as epidemiological and cohort evidence predict that the level of glucose control impacts cardiovascular events (22). However, prospective clinical trials have demonstrated that current strategies for improving blood glucose do not usually achieve this goal, but rather obtaining a period of optimal control may confer long-term CVD benefits (22). Therefore, large prospective follow-up studies are needed to further evaluate the predictive value of impaired FPG for forecasting CAD events and the cardiovascular prognosis.

The effects of hyperglycemia on cardiovascular risks are independent of any other known risk factors, although no data from primary interventional trials are yet available (23). The pathogenic effects of hyperglycemia on the blood vessel wall are already present in the early stages of glucose intolerance (23). A high glucose level activates a proatherogenic phenotype in all cell types in the vessel wall, including endothelial cells, vascular smooth muscle cells, inflammatory cells, fibroblasts and platelets, leading to a feedforward atherogenic response (22). In addition, the mechanisms underlying endothelial dysfunction in patients with diabetes in- 
clude altered glucose metabolism, impaired insulin signaling, low-grade inflammation and increased reactive oxygen species generation (24). Furthermore, alterations in the differentiated state of vascular smooth muscle cells play a critical role in the pathogenesis of atherosclerosis (25), and several lines of evidence highlight the relevance of glucose metabolism for regulating vascular smooth muscle cell (VSMC) proliferation, indicating a new area for exploration with respect to controlling the vascular pathogenesis (25).

This study is associated with several limitations. First, all subjects were candidates undergoing coronary angiography. Therefore, our results may have been subject to subject selection bias and may not be applicable to the general population. Second, this was a cross-sectional study and we did not evaluate the duration of impaired FPG or T2DM, which may be an important factor affecting the SYNTAX score. Importantly, the subjects with known T2DM may have had a longer disease duration than those with unknown T2DM, and we were unable to define the duration of previously unknown T2DM, nor did we analyze the effects of the duration of T2DM in our model. Moreover, the adjusted odds ratio does not specifically reflect the effects of the duration of known T2DM. We are unable to explain why this contributed to a slightly higher adjusted odds ratio in the subjects with unknown T2DM compared with that observed in the subjects with known T2DM. Third, all of the subjects were Chinese.

In conclusion, Chinese subjects with impaired FPG have a significantly elevated risk of having an intermediate or high SYNTAX score, indicating the risk of severe coronary artery lesions. Subjects with known or previously unknown T2DM may have severe coronary artery lesions. These findings suggest the importance of achieving better glycemic control in order to prevent coronary atherosclerosis and improve the cardiovascular prognosis.

\section{The authors state that they have no Conflict of Interest (COI).}

\section{Financial Support}

This work was supported by an Academic Progression Grant from Luoyang Central Hospital Affiliated with Zhengzhou University.

\section{Acknowledgement}

We thank Zhenghao Liu and Lingling Pan for their help in collecting the data during the revision period.

Xishan Yang and Hui Liu contributed equally to this work.

\section{References}

1. Warraich HJ, Nasir K. Subclinical cardiovascular disease assessment in persons with diabetes. Curr Cardiol Rep 15: 358, 2013.

2. Davidson $\mathrm{MH}$. Cardiovascular risk factors in a patient with diabetes mellitus and coronary artery disease: therapeutic approaches to improve outcomes: perspectives of a preventive cardiologist. Am J Cardiol 110: 43B-49B, 2012.
3. Hanna-Moussa A, Gardner MJ, Kurukulasuriya LR, Sowers JR. Dysglycemia/prediabetes and cardiovascular risk factors. Rev Cardiovasc Med 10: 202-208, 2009.

4. Schinner S, Füth R, Kempf K, et al. A progressive increase in cardiovascular risk assessed by coronary angiography in non-diabetic patients at sub-diabetic glucose levels. Cardiovasc Diabetol 10: 56, 2011.

5. Zornitzki T, Ayzenberg O, Gandelman G, et al. Diabetes, but not the metabolic syndrome, predicts the severity and extent of coronary artery disease in women. QJM 100: 575-581, 2007.

6. Berry C, Noble S, Grégoire JC, et al. Glycaemic status influences the nature and severity of coronary artery disease. Diabetologia 53: 652-658, 2010.

7. Yan Q, Gu WQ, Hong J, et al. Coronary angiographic studies of impaired glucose regulation and coronary artery disease in Chinese nondiabetic subjects. Endocrine 36: 457-463, 2009.

8. Yadav M, Palmerini T, Caixeta A, et al. Prediction of coronary risk by SYNTAX and derived scores: synergy between percutaneous coronary intervention with taxus and cardiac surgery. J Am Coll Cardiol 62: 1219-1230, 2013.

9. Ikeda N, Iijima R, Hara H, Moroi M, Nakamura M, Sugi K. Glycated hemoglobin is associated with the complexity of coronary artery disease, even in non-diabetic adults. J Atheroscler Thromb 19: 1066-1072, 2012.

10. Langenfeld MR, Forst T, Hohberg C, et al. Pioglitazone decreases carotid intima-media thickness independently of glycemic control in patients with type 2 diabetes mellitus: results from a controlled randomized study. Circulation 111: 2525-2531, 2005.

11. Lee CL, Li TC, Lin SY, et al. Dynamic and dual effects of glycated hemoglobin on estimated glomerular filtration rate in type 2 diabetic outpatients. Am J Nephrol 38: 19-26, 2013.

12. Park C, Guallar E, Linton JA, et al. Fasting glucose level and the risk of incident atherosclerotic cardiovascular diseases. Diabetes Care 36: 1988-1993, 2013.

13. Shaye K, Amir T, Shlomo S, Yechezkel S. Fasting glucose levels within the high normal range predict cardiovascular outcome. Am Heart J 164: 111-116, 2012.

14. Sarwar N, Aspelund T, Eiriksdottir G, et al. Markers of dysglycaemia and risk of coronary heart disease in people without diabetes: Reykjavik prospective study and systematic review. PLoS Med 7: e1000278, 2010.

15. Saito I. Epidemiological evidence of type 2 diabetes mellitus, metabolic syndrome, and cardiovascular disease in Japan. Circ J 76: 1066-1073, 2012.

16. Bhat SL, Abbasi FA, Blasey C, Reaven GM, Kim SH. Beyond fasting plasma glucose: the association between coronary heart disease risk and postprandial glucose, postprandial insulin and insulin resistance in healthy, nondiabetic adults. Metabolism 62: 1223-1226, 2013.

17. Laukkanen JA, Mäkikallio TH, Ronkainen K, Karppi J, Kurl S. Impaired fasting plasma glucose and type 2 diabetes are related to the risk of out-of-hospital sudden cardiac death and all-cause mortality. Diabetes Care 36: 1166-1171, 2013.

18. Van de Veire NR, De Winter O, Gir M, De Buyzere M, Van de Wiele C, De Sutter J. Fasting blood glucose levels are related to exercise capacity in patients with coronary artery disease. Am Heart J 152: 486-492, 2006.

19. Arbel Y, Zlotnik M, Halkin A, et al. Admission glucose, fasting glucose, HbA1c levels and the SYNTAX score in non-diabetic patients undergoing coronary angiography. Clin Res Cardiol 103: 223-227, 2014.

20. Wang JS, Lee IT, Lee WJ, et al. Performance of HbA1c and fasting plasma glucose in screening for diabetes in patients undergoing coronary angiography. Diabetes Care 36: 1138-1140, 2013.

21. Doerr R, Hoffmann U, Otter W, et al. Oral glucose tolerance test and $\mathrm{HbA}_{1} \mathrm{c}$ for diagnosis of diabetes in patients undergoing coro- 
nary angiography: [corrected] the Silent Diabetes Study. Diabetologia 54: 2923-2930, 2011.

22. Reusch JE, Wang CC. Cardiovascular disease in diabetes: where does glucose fit in? J Clin Endocrinol Metab 96: 2367-2376, 2011.

23. Milicevic Z, Raz I, Beattie SD, et al. Natural history of cardiovascular disease in patients with diabetes: role of hyperglycemia. Dia- betes Care 31 (Suppl 2): S155-S160, 2008.

24. Sena CM, Pereira AM, Seiça R. Endothelial dysfunction - a major mediator of diabetic vascular disease. Biochim Biophys Acta 1832: 2216-2231, 2013.

25. Chiong M, Morales $\mathrm{P}$, Torres $\mathrm{G}$, et al. Influence of glucose metabolism on vascular smooth muscle cell proliferation. Vasa 42: 816, 2013.

(C) 2015 The Japanese Society of Internal Medicine http://www.naika.or.jp/imonline/index.html 